\title{
Dust and cloud detection at the Mars limb with UV scattered sunlight with SPICAM
}

\author{
P. Rannou, ${ }^{1}$ S. Perrier, ${ }^{1}$ J.-L. Bertaux, ${ }^{1}$ F. Montmessin, ${ }^{1}$ O. Korablev, ${ }^{2}$ and A. Rébérac ${ }^{1}$ \\ Received 1 February 2006; revised 7 April 2006; accepted 5 April 2006; published 30 September 2006. \\ [1] The UV detector of Spectroscopy for the Investigation of the Characteristics of the \\ Atmosphere of Mars (SPICAM) on board Mars Express has measured several profiles of \\ light scattered at the limb of Mars. In this paper we present 33 profiles taken between \\ January 2004 and August 2005. Scattering of UV light at the limb of Mars is due to \\ the molecules of the atmosphere, dust particles, and sometimes cloud particles which \\ appear as detached layers above the extended dust layer. We have used a radiative transfer \\ model to retrieve the haze and cloud properties. Rough estimate of the particle size shows \\ that both cloud particles and dust particles above $20 \mathrm{~km}$ are in the range 10 to $100 \mathrm{~nm}$. \\ Such particles are much smaller than micron-sized dust particles previously observed \\ in the lower atmosphere, generally from landers. Gravitational segregation is thought to be \\ responsible for these differences in particle size between low and high atmosphere.
}

Citation: Rannou, P., S. Perrier, J.-L. Bertaux, F. Montmessin, O. Korablev, and A. Rébérac (2006), Dust and cloud detection at the Mars limb with UV scattered sunlight with SPICAM, J. Geophys. Res., 111, E09S10, doi:10.1029/2006JE002693.

\section{Introduction}

[2] Mars climate is controlled on one hand by the dust content of the atmosphere and the clouds at visible wavelengths, and on the other hand, by the gaseous components in thermal infrared and the solid state component again: dust and clouds. Several years of monitoring of Mars climate have shown a strong year-to-year variability in dust loading, and therefore in the climate. In the three last decades, the observation of Mars has slowly shifted from a phase where the purpose was to get the main characteristics of the various atmospheric components and to understand the main structures [e.g., Pollack et al., 1979], to a phase where the main components (dust, water content, clouds) are permanently monitored [e.g., Smith, 2004]. This survey of Mars is of great interest because it is developed along with climate models. After the Earth, Mars is the first planet to receive such attention.

[3] The global monitoring of aerosol component by MGS TES [Smith, 2004] consists in measurement of total optical depth of mineral dust and ice clouds in the thermal IR range (9 $\mu \mathrm{m}$ and $12 \mu \mathrm{m}$ consequently). The information on the aerosol properties, constraining the size of particles and their distribution is available mostly from sky images at different visible wavelengths taken from the surface [e.g., Pollack et al., 1995; Tomasko et al., 1999] and from orbiter observations of a surface target taken at different phase angles, frequently designated as Emission Phase Functions, EPF, after Viking IRTM measurements [Clancy et al., 1995]. Such measurements were also performed with

\footnotetext{
${ }^{1}$ Service d'Aéronomie du CNRS, Université de Versailles St-Quentin, Verrières le Buisson, France.

${ }^{2}$ Space Research Institute (IKI), Moscow, Russia.

Copyright 2006 by the American Geophysical Union. 0148-0227/06/2006JE002693
}

MGS TES [Clancy et al., 2003], and with Mars Express spectral instruments (the results are not published yet). Generally admitted model of Martian dust from such measurements is consistent with $1-2 \mu \mathrm{m}$ sized particles, the size distribution being rather poorly constrained. It should be noted that the most reliable estimations result either from measurements made from the ground of Mars, and represent therefore the dust properties of the lower atmosphere, or are available from remote measurements in the near-IR and the IR spectral ranges.

[4] Global monitoring is often performed in nadir viewing and, although it produces an exhaustive spatio-temporal coverage of the annual cycle, it often misses the vertical dimension. In the past, few measurements have given a vertical view of the dust and the cloud layer at visible or at infrared wavelengths. Some detached layers were discovered at Viking camera images [Jaquin et al., 1986]. During Phobos-88 Soviet mission several solar occultations allowed to study the aerosol vertical structure [Chassefière et al., 1992; Korablev et al., 1993]. Unfortunately more recent MGS MOC limb images, and TES limb data has not been analyzed yet with the aim to retrieve aerosol vertical structure. A common problem is the determination of the absolute altitude on the limb. Because limb viewings are generally sporadic, they hardly provide a complete view of the planet but rather give hints of how the vertical structure could look like from some selected cases. A significant advantage of such limb viewing is to allow the detection of detached layers, generally due to clouds, with a good altitude resolution.

[5] Some remote measurements performed in the UV and the visible spectral ranges have suggested much smaller particles. UVS measurements from Mariner 9 were interpreted with 60-200 nm particles [Pang and Ajello, 1977; Chýlek and Gramm, 1978]. Small, weakly absorbing par- 
ticles were systematically retrieved from ground-based polarimetry observations [Morozhenko, 1974; Dollfus et al., 1974]. Recently, a set of such measurements taken in seventies was reinterpreted taking into account non-spherical particles to find particles size as small as $40-80 \mathrm{~nm}$ [Dlugach et al., 2002]. These measurements have received, however, a limited credit because their interpretation strongly depends on a priori assumptions. Some of Phobos-88 occultation measurements [Chassefière et al., 1992] performed at few wavelengths between 270 and $930 \mathrm{~nm}$ have revealed detached cloud layers with particle size of $0.3-$ $0.65 \mu \mathrm{m}$.

[6] SPICAM on board Mars Express/ESA orbiter has performed a series of limb observations using occultation techniques and limb scattering photometric observations the UV and near-IR spectral range, allowing somewhat systematical study of Mars aerosols vertical structure. The retrieval method for the occultation technique is presented in a companion paper [Quémerais et al., 2006], while the results for dust and clouds are discussed by Montmessin et al. [2006b]. In this paper, we present the observations and the analysis of UV solar light scattered at the limb of Mars made by the UV spectrometer of SPICAM. We first display the intensity profiles, and we discuss some aspects of the data. We then describe the procedure used to analyze the observations and finally, we discus the results and the uncertainties related to the retrieval procedure.

\section{Presentation of the Data}

[7] SPICAM is a spectrometer which makes a part of its observations in the UV range, between 118 and $320 \mathrm{~nm}$. Light is collected by a parabolic mirror, reflected onto a spectrometer through a slit. The light is separated along one dimension of the CCD array $(384 \times 288$ active pixels $)$ and recorded with a frequency of $1 \mathrm{~Hz}$. The other dimension of the CCD allows spatial resolution. When observing at bright limb, the field of view (FOV) of SPICAM UV is determined by the width of the narrow part of the slit (2 pixels of the CCD) and the height of each of five spectral bands (usually, 4 pixel lines of the CCD), and is therefore rather small: $\mathrm{FOV}=0.02^{\circ} \times 0.04^{\circ}$ (see more in general presentation of the instrument by Bertaux et al. [2006]. The vertical resolution on the limb is determined by the spacecraft-tolimb distance or altitude (see below), and is different for different observations. Some pixels at one end of the CCD are masked to provide the dark current measurement and the electronic offset. The data are digitized in 4096 levels. Several observation modes are possible, in nadir and limb viewing. A detailed description is given by Bertaux et al. [2000, 2005, 2006].

[8] To perform limb measurements, Mars Express spacecraft points the line of sight (LOS) of SPICAM (and other remote instruments, such as OMEGA) toward limb of Mars and remains in an inertial attitude. The orbital motion of the spacecraft results in vertical or oblique scan of the limb. Sometimes the LOS intersects the bright limb twice, providing both the ingress and egress profiles on a single orbit. The SPICAM UV spectrometer observes stars to perform stellar occultation; thanks to it the LOS direction is well determined in the coordinates of the spacecraft. The position of the true limb (the edge of the solid body of the planet) and the altitude of a limb observation are therefore also known with high accuracy ( \pm FOV).

[9] When considering scattering in general, and especially limb scattering, it is of the greatest importance to have a good absolute calibration allowing to derive intensities relative to the solar flux, as the radiance factor, to study the properties of the atmosphere. The conversion between digitized numbers and the real flux observed at Mars comes from the observation of calibrated stars (for details, see Bertaux et al. [2006]). One noticeable restriction when using the sun as a source of light is the weakness of the flux emitted at wavelength below about $180 \mathrm{~nm}$ compared to wavelength above $250 \mathrm{~nm}$. It turns out that SPICAM does not detect solar light from wavelengths shorter than $180 \mathrm{~nm}$ and only a faint light between 180 and about $220 \mathrm{~nm}$. The product of SPICAM that we are going to analyze here is the radiance factor $(\mathrm{I} / \mathrm{F})$ observed at Mars limb, between 220 and $300 \mathrm{~nm}$.

\subsection{Selection of the Profiles}

[10] We are interested by measurements of light scattered at Mars limb, that is, above the radius of the solid body of Mars. Between 9 January 2004 and 15 August 2005, SPICAM has made 1755 different observations including observations during the commissioning phase. Only 60 observations are dedicated to the limb with at least six points between the solid body and $60 \mathrm{~km}$ altitude, and with a solar zenith angle, at the limb, lower than $100^{\circ}$. Depending on the observing configuration, SPICAM limb observation sometimes suffers from stray light. The slit which is used when observing extended sources has a width of $50 \mathrm{~nm}$, which corresponds $1.1 \mathrm{~nm}$ of resolution, for the lower part of the CCD. It is 10 times larger for the other part. In principle, the light incoming on a given line of CCD should not affect the others, but this is not the case. Moreover, with some orientation of SPICAM, the wider part of the slit is illuminated by the bright Mars disk whereas the narrower part of the slit is probing a fainter part of limb. In this special case, even a moderate part of light lost from the wide part of the slit can significantly affect another part of the CDD, probing a fainter part of the limb through the narrow part of the slit. A procedure is under work to correct this problem, but in this paper we only work with data shown to be not affected. To discard flawed profiles, we use the fact that stray light has a continue spectral behavior which do not matches the marked features of the solar spectrum. The stray light offsets the observed intensity reflected by the atmosphere and dims the contrasts due to the solar spectral features. Then, it produces the correlated structures on the radiance factor $I / F$. Radiance factors due to dust, clouds and gas have simple spectral behaviors modeled with a power law (as shown further). We fit the spectral behavior of with first- and a second-order polynomials, and used a $\chi^{2}$ test to decide if the spectral behavior departs from a first-order polynomial. We only keep the profiles where absolutely no stray light is detected. Only 27 measurements, producing 33 different profiles (some observations cross Mars limb twice) can be studied in the frame of this work (Figure 1). The geometry and the date of each observation are reported in Table 1. The altitude is quoted in this paper is defined as follows. The Mars nearest point along the line of sight is at a distance $d$ from the center of Mars. The 

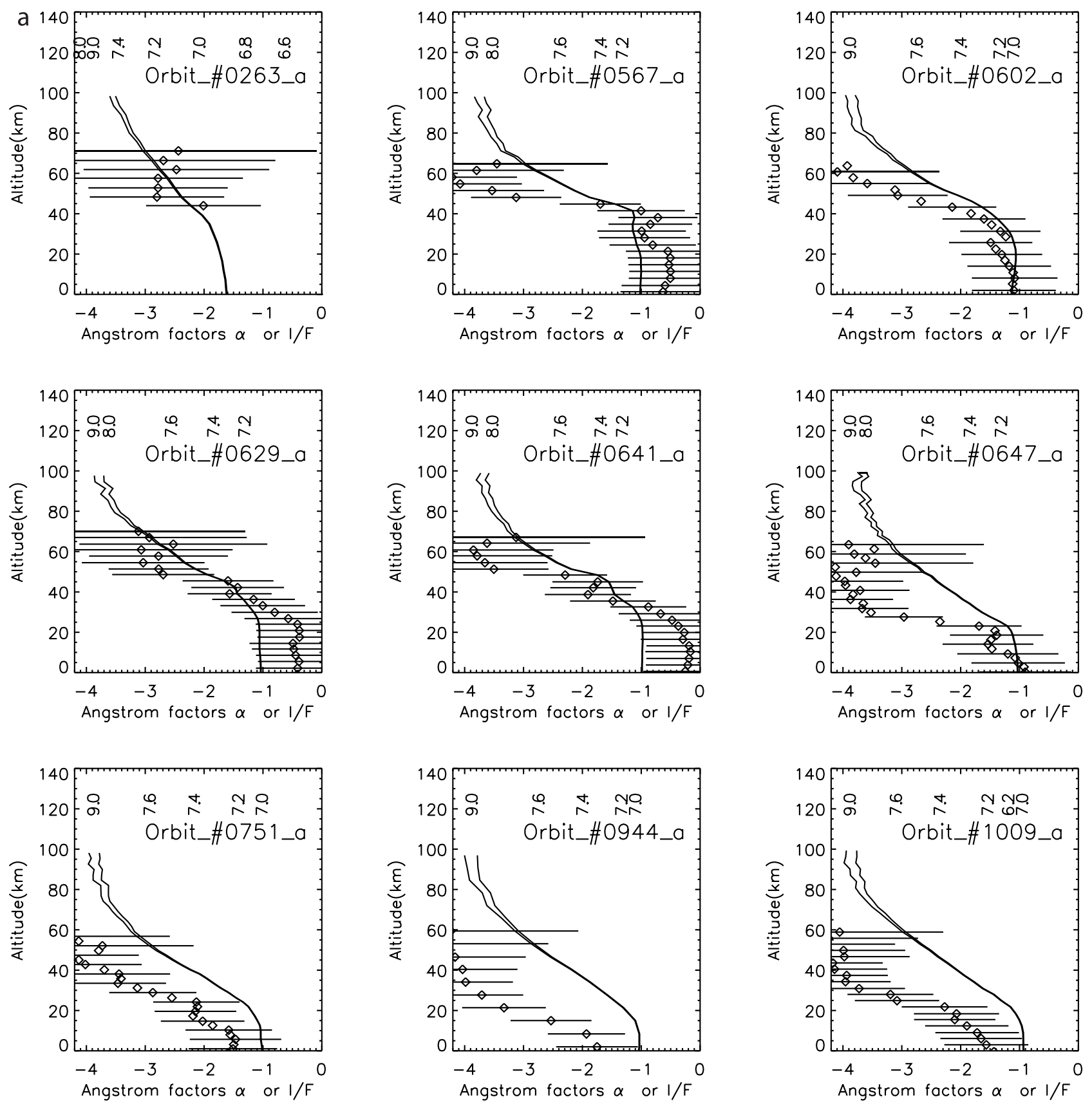

Figure 1. (a) Radiance factors $\mathrm{I} / \mathrm{F}$ at $250 \mathrm{~nm}$ (the two solid lines show the radiance factor \pm the uncertainties on $\mathrm{I} / \mathrm{F}$ ) and Angström factors $\alpha$ as defined in equation (3) (diamonds with error bars) for several orbits. Orbit numbers are noted with ${ }_{a}$ or ${ }_{b}$, since the limb is crossed twice for some orbits. The numbers at the top of each plot show the correspondence between the radius of particles (here noted as $-\log _{10}$ (radius) with the radius in meters) and the Angström factors $\alpha$ on abscissae. This relation only stands for the top of the profile, where the atmosphere tangential opacity is low. (b) Same as Figure 1a.

(c) Same as Figure 1a. (d) Same as Figure 1a.

latitude of this point defines the local radius $R_{L}$ of the Mars ellipsoid reference used by SPICE (IAU), with $R_{e q}=$ $3396.19 \mathrm{~km}$ at the equator and $R_{p o l}=3376.20 \mathrm{~km}$ at the poles. The altitude is given as $z=d-R_{L}$.

\subsection{Description of the Limb Profiles}

[11] The data we are using in this paper is under the form of vertical profiles of the scattered light, taken at 146 different wavelengths between 220 and $300 \mathrm{~nm}$, with a sampling of about $0.5 \mathrm{~nm}$ and a resolution of $1.5 \mathrm{~nm}$. At these wavelengths, all the scatterers (gas molecules and submicron- or micron-sized particles) have simple behaviors which may be modeled by a simple relation between extinction or scattering coefficients and wavelength, as

$$
\beta(\lambda)=\beta_{0}\left(\frac{\lambda}{\lambda_{0}}\right)^{\alpha}
$$



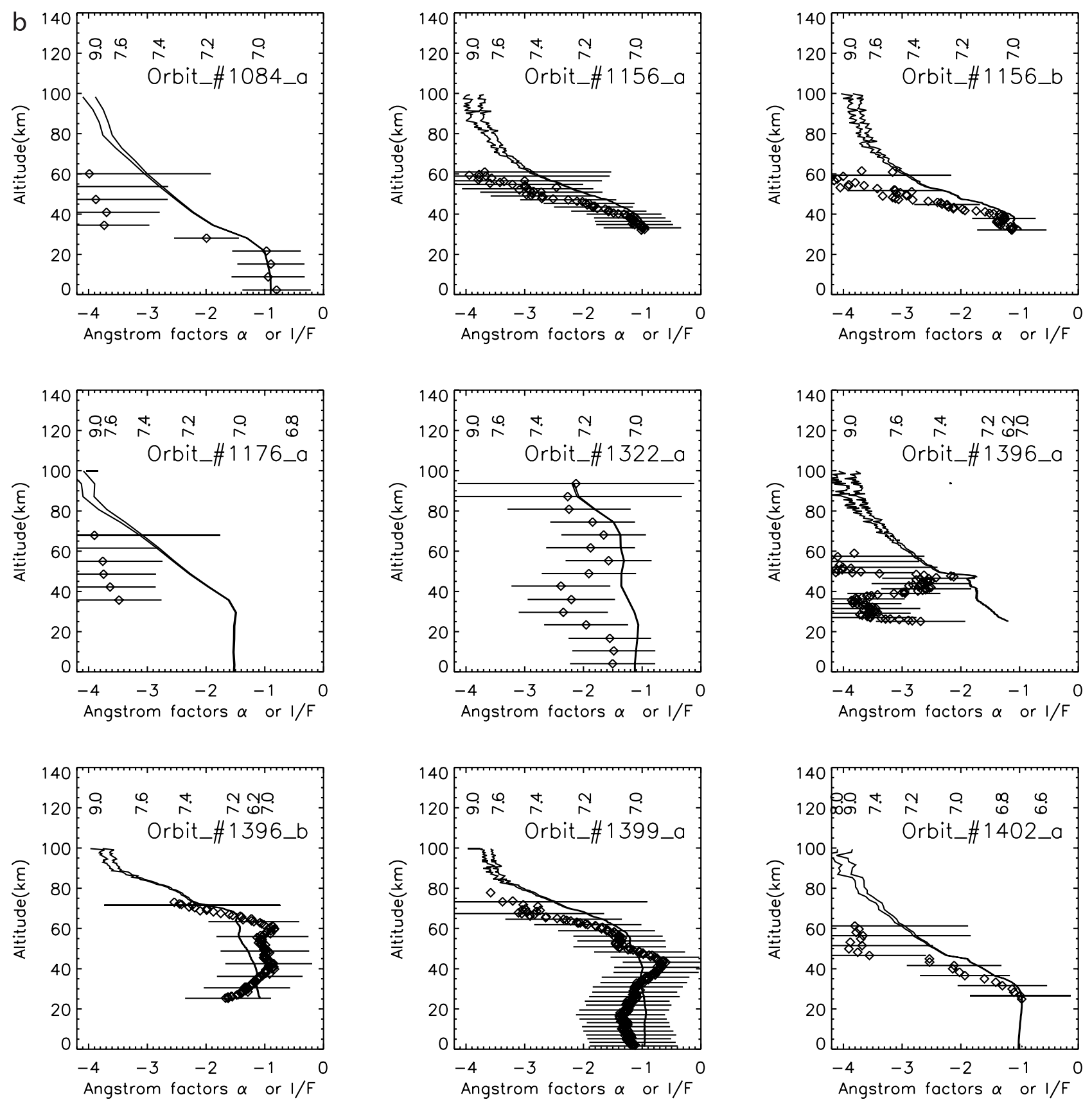

Figure 1. (continued)

where $\beta(\lambda)$ is, for instance, the extinction coefficient, $\lambda$ the wavelength and $\alpha$ the Angström factor. Note that we have adopted a convention for $\alpha$ which gives the opposite value of those of Montmessin et al. [2006b]. In the same way, the radiance factor shows the same type of behavior as a function of the wavelength. This enables to display the data in a very concise way, the radiance factor as a function of the altitude at a given wavelength (e.g., $250 \mathrm{~nm}$ ) and the Angström factor (Figure 1) defined as

$$
\frac{I}{F}(\lambda)=\frac{I}{F}\left(\lambda_{0}\right)\left(\frac{\lambda}{\lambda_{0}}\right)^{\alpha}
$$

which may be written as well:

$$
\log \left(\frac{I / F(\lambda)}{I / F\left(\lambda_{0}\right)}\right)=\alpha \log \left(\frac{\lambda}{\lambda_{0}}\right)
$$

[12] The upper parts of the profiles generally have an Angström factor of $\alpha \simeq-4$ which is a characteristics of the Rayleigh scattering by the atmosphere. The dust and cloud particles can be detected easily because they produce structures in the radiance factor profile, and these layers are even more visible in the Angström factor profile. For 

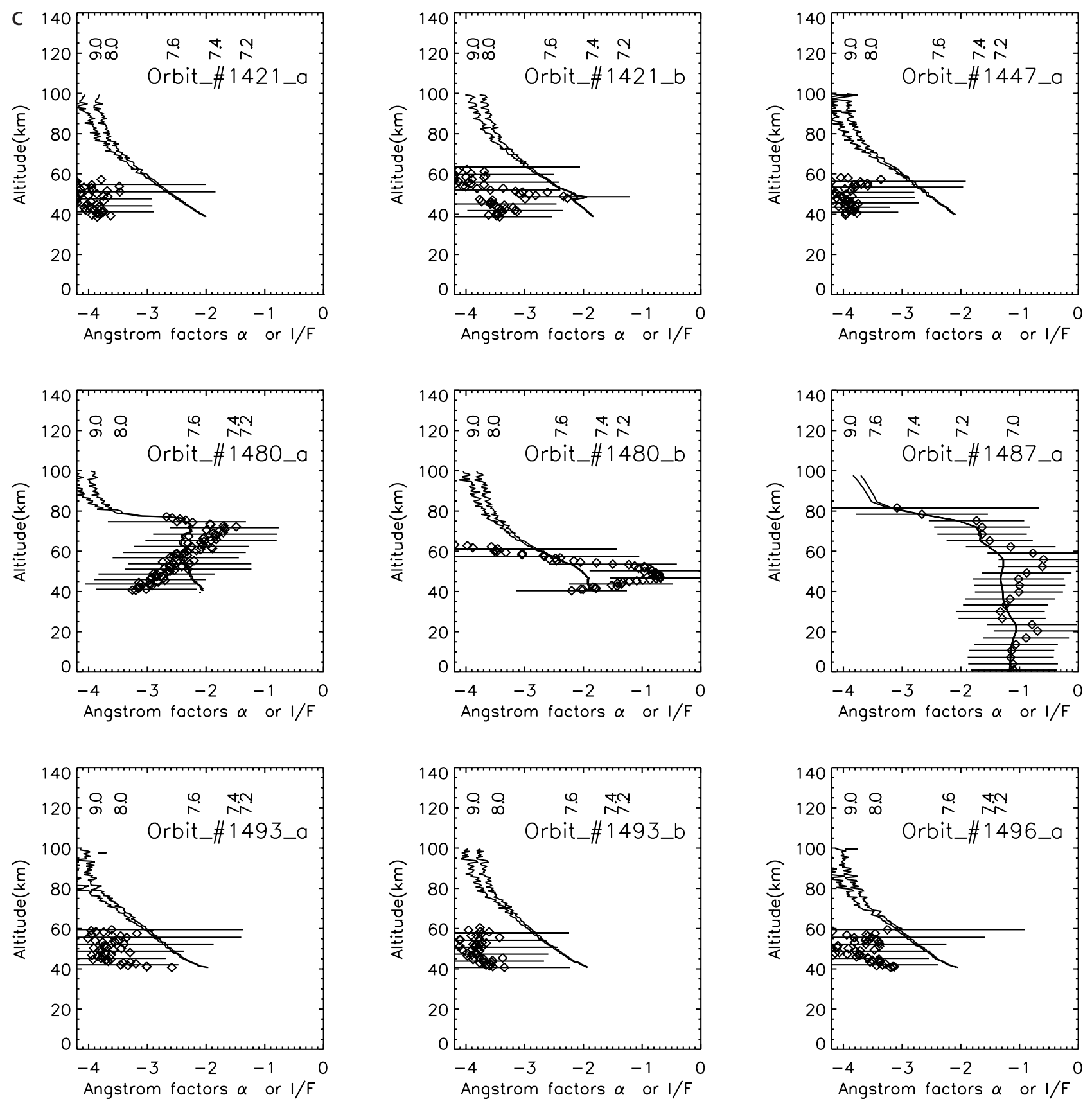

Figure 1. (continued)

some of the profiles, the lower part of the atmosphere (generally below $20 \mathrm{~km}$ ) have a spectral behavior that does not match the simple law of equation (3), but need a second term in $\alpha^{\prime} \log ^{2}\left(\lambda \lambda_{0}\right)$. This is the case of the profiles $263 a$, $1176 a$ and $1402 a$. Ozone is probably responsible for this behavior, and for the purpose of a first analysis, we do not consider these parts of the profiles.

\section{Procedure of the Analysis}

\subsection{Model of Light Scattering at the Limb}

[13] The intensity scattered at the limb is the integral of the scattered light by each part of the atmosphere along the line of sight. The elementary scattered intensity is

$$
d\left(\frac{I}{F}\right)=\frac{1}{4} \beta \omega P\left(\theta_{s}\right) e^{-\tau_{1}(x)-\tau_{0}(x)} d x
$$

where $x$ is the location on the axis of the line of sight, and $x=0$ is taken in the plane of the limb. $\beta$ and $\omega$ are the extinction coefficient and mean single scattering albedo of the atmosphere at $x$, and $P\left(\theta_{S}\right)$ is the mean phase function of the atmosphere at $x$ for the scattering angle $\theta_{s} . \tau_{1}(x)$ is the optical depth between the location $x$ and the observer 

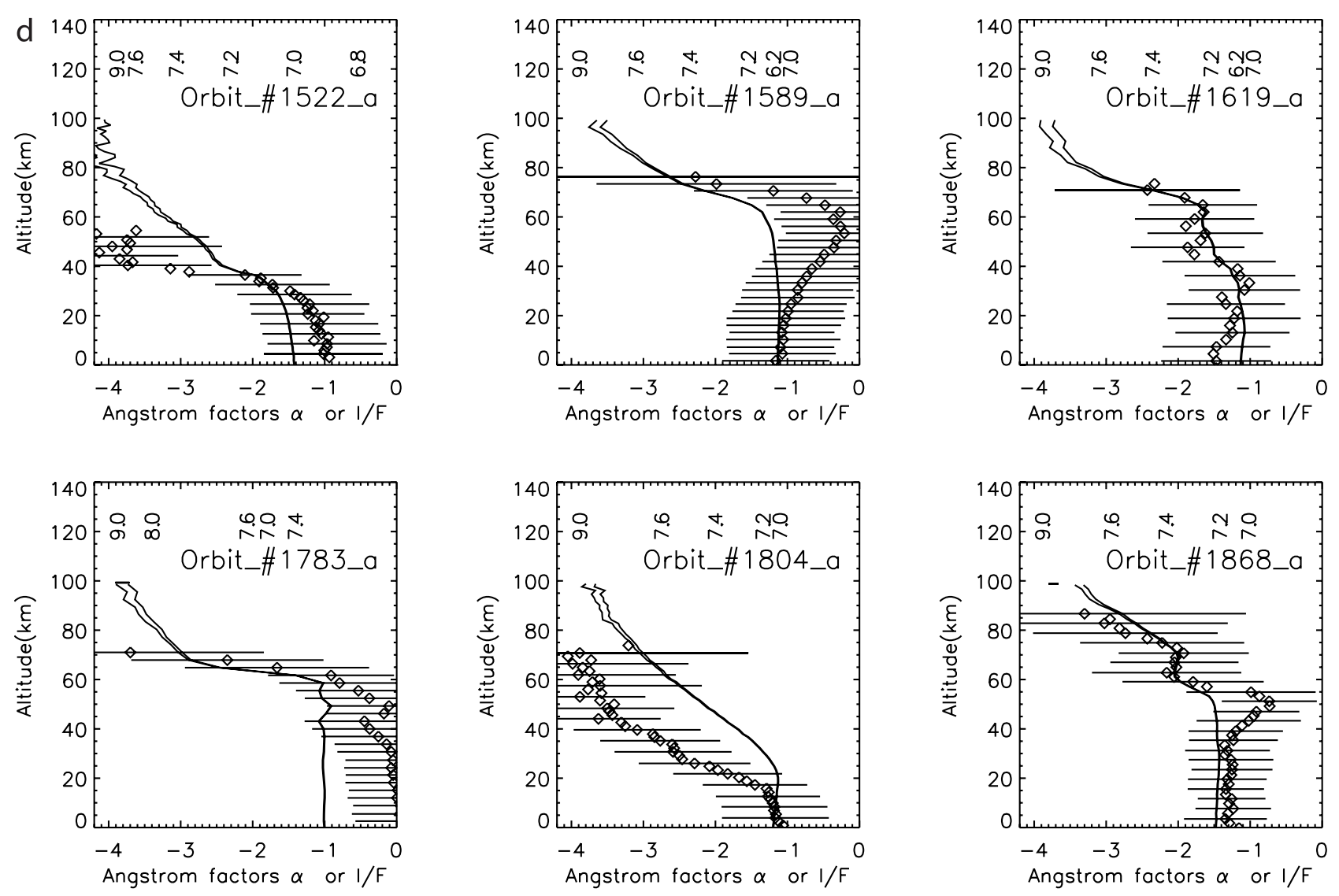

Figure 1. (continued)

(emerging path after the scattering) and $\tau_{0}(x)$ the optical depth between the sun and the location $x$ (incident path before scattering). By convention, the integral of the phase function over the space is set to $4 \pi$, and we have written the solar flux outside the atmosphere $\pi \times F$. The total radiance factor seen from outside the atmosphere is integrated over the whole $\mathrm{x}$ axis.

$$
I / F(z)=\int_{-\infty}^{\infty} \frac{d I / F}{d x} d x
$$

[14] The main difficulty is to compute the terms $\tau_{1}(x)$ and $\tau_{0}(x)$ from geometrical considerations. Equation (4) stands for single scattering only. To account for the multiple scattering, one should account for the internal intensity field $I(\theta, \phi)$ as well as the direct solar flux. Evaluating the multiple scattering is a delicate matter. One difficulty is that multiple scattering in one location involves the whole atmosphere. To retrieve atmospheric properties from photometric observations, one need to know the properties of all the atmosphere. This can be achieved using a onion peeling procedure, with an iterative procedure [Jaquin et al., 1986]. This necessitates a model where the intensity field can be computed accurately, even in the case of multiple scattering. This also implies that the retrieval procedure can be tracted down to the surface. A simplest way is to compute the single scattering solution with equations (4) and (5), and to estimate the importance of the multiple scattering with a simple model (e.g., two-stream model in parallel plane geometry). This technique is convenient when the atmosphere is poorly known [Rages and Pollack, 1983], or when the retrieval procedure does not enable to retrieve the complete atmosphere, which prevents any iterative procedure.

[15] For this work, we have used a model which accounts for the single scattering in a spherical atmosphere [Rages and Pollack, 1983; Rannou et al., 1997]. The multiple scattering is estimated in a very simple way by enhancing the scattered intensity by a single factor $\rho$ which is the ratio of the multiple scattering and the single scattering inside the atmosphere. To estimate the value of $\rho$, we have used a two stream model, and we have used dust characteristics found in literature [Markiewicz et al., 1999] to create a model of atmosphere properties. We find that $\rho$ is constant with altitude in regions where the normal optical depth is much smaller that unity (which occurs between 20 and $60 \mathrm{~km}$ depending on the dust loading). These layers are those for which the retrieval is possible. In these case, we know that the multiple scattered light essentially comes from the solid planet and the layers below the level we consider. We estimates the ratio $\rho$ to about 1.2 in average, for the range of solar zenith angle we meet in this work. At this stage, we only use this first-order correction since we have no information about the lower part of the atmosphere. We have recently performed tests between this model and a Monte-Carlo model developed for a dust sensor [after Tran 
Table 1. Census of the Limb Profiles by SPICAM

\begin{tabular}{|c|c|c|c|c|c|c|}
\hline Orbit & Phase & Sza & Latitude & Longitude & Local Time & Date \\
\hline 0263 _a & 14.6 & 93.1 & 62.4 & 137.5 & 4.8 & 2004-04-02 \\
\hline 0567_a & 103.8 & 51.5 & 16.4 & 225.7 & 8.4 & 2004-06-30 \\
\hline $0602^{-} \mathrm{a}$ & 79.9 & 77.3 & 26.5 & 357.2 & 6.3 & $2004-07-10$ \\
\hline 0629 a & 89.9 & 76.7 & 21.4 & 229.8 & 6.4 & 2004-07-17 \\
\hline $0641^{-} \mathrm{a}$ & 99.6 & 62.1 & 29.5 & 145.6 & 7.4 & $2004-07-21$ \\
\hline 0647_a & 92.2 & 60.8 & 59.5 & 298.2 & 7.3 & $2004-07-22$ \\
\hline $0751^{-} \mathrm{a}$ & 71.4 & 72.7 & 44.7 & 152.3 & 6.0 & $2004-08-21$ \\
\hline 0944_a & 78.1 & 54.3 & 70.6 & 68.9 & 15.4 & $2004-10-14$ \\
\hline $1009^{-} \mathrm{a}$ & 59.5 & 60.2 & 79.4 & 174.5 & 15.5 & $2004-11-01$ \\
\hline 1084_a & 41.5 & 73.0 & 80.1 & 206.8 & 4.1 & $2004-11-22$ \\
\hline 1156 a & 38.7 & 50.7 & 32.5 & 154.4 & 15.7 & $2004-12-12$ \\
\hline $1156 \mathrm{~b}$ & 36.1 & 53.5 & 1.6 & 149.1 & 15.4 & $2004-12-12$ \\
\hline $1176^{-} \mathrm{a}$ & 26.4 & 88.2 & -66.2 & & 14.1 & $2004-12-18$ \\
\hline $1322^{-} \mathrm{a}$ & 35.8 & 51.9 & -39.1 & 17 & 12.7 & $2005-01-27$ \\
\hline 1396_a & 60.5 & 47.8 & 50.1 & 333.2 & 13.8 & $2005-02-17$ \\
\hline $1396 \mathrm{~b}$ & 58.9 & 29.7 & 17.8 & 333.5 & 13.9 & $2005-02-17$ \\
\hline 1399_a & 43.9 & 47.8 & 20.0 & 321.5 & 8.8 & $2005-02-18$ \\
\hline $1402^{-} \mathrm{a}$ & 11.1 & 83.0 & 86.6 & 345.7 & 5.8 & $2005-02-19$ \\
\hline 1421_a & 91.1 & 73.1 & 68.3 & 73.8 & 15.9 & 2005-02-24 \\
\hline 1421_b & 88.7 & 42.4 & 45.8 & 31.0 & 13.2 & $2005-02-24$ \\
\hline 1447_a & 97.4 & 77.6 & 69.5 & 49.7 & 16.4 & $2005-03-03$ \\
\hline 1480_a & 105.0 & 82.9 & 69.7 & 60.8 & 17.0 & $2005-03-13$ \\
\hline $1480-\mathrm{b}$ & 102.8 & 53.6 & 54.6 & & 13.0 & $2005-03-13$ \\
\hline 1487_a & 40.4 & 56.4 & -50.3 & 345.8 & 10.3 & $2005-03-15$ \\
\hline $1493^{-} \mathrm{a}$ & 108.0 & 84.9 & 69.3 & 228.7 & 17.3 & $2005-03-16$ \\
\hline $1493 \_b$ & 105.9 & 56.2 & 56.4 & 161.7 & 12.9 & $2005-03-16$ \\
\hline $1496^{-} \mathrm{a}$ & 109.3 & 85.6 & 69.1 & 295.9 & 17.4 & $2005-03-17$ \\
\hline 1522 a & 27.8 & 92.7 & 86.5 & 51.3 & 3.3 & 2005-03-24 \\
\hline 1589_a & 57.1 & 64.4 & -22.0 & 16.7 & 7.7 & 2005-04-12 \\
\hline 1619 a & 58.4 & 60.9 & -51.4 & 322.8 & 8.6 & $2005-04-21$ \\
\hline 1783 a & 131.4 & 69.3 & -48.2 & 222.7 & 17.2 & 2005-06-06 \\
\hline 1804_a & 79.6 & 67.6 & -86.0 & 192.9 & 10.7 & $2005-06-11$ \\
\hline 1868 a & 66.2 & 84.7 & -9.6 & 16.1 & 6.1 & 2005-06-29 \\
\hline
\end{tabular}

and Rannou, 2004]. These tests show that the simple model presented here capture the essential behaviors of the limb scattering process, and reaches accuracies of $20 \%$. Clearly, in this work, the multiple scattering factor $\rho$ is less constrained than in the work of Tran and Rannou [2004]. However, we will see that this does not affect much the retrieval of the particle radius and the concentration of particles (and then the scaled scattering coefficient $\beta \omega P(\theta))$ which differ by less than a factor of 2 when $\rho$ is varied.

\subsection{Retrieval Procedure}

[16] The retrieval procedure used here is simple. We used a kind of onion peeling inversion, where the free parameters are the effective radius $r_{\text {eff }}$ of the particle distribution, assumed to follow a log-normal distribution, and the concentration of particles $n$. The effective variance is set to $v_{\text {eff }}=0.3$ which is a standard value for Martian dust distribution [Markiewicz et al., 1999]. The atmosphere is discretized in several spherical layers where the distribution is supposed to be homogeneous. In each layer $i$, we define the effective radius and the concentration of particle as $r_{e f f}^{(i)}$ and $n^{(i)}$. The $I / F$ profile is also discretized and each line of sight $(i)$ crosses the limb plane at an altitude which corresponds to the altitude of the lower bound of the layer $i$ (that is the level $i$ ). The radiance factor $I / F^{(i)}$ at the level $i$ depends on the particle distribution effective radius and concentration of any layer between the layer $i$ and the top of the atmosphere. With the onion peeling procedure, and assuming only single scattering, the characteristics of the layer above $i$ (that is $i+$ $1, i+2, \ldots)$ are supposed to be known. Then, the radiance factor at the level $i$ only depends on the unknowns $r_{e f f}^{(i)}$ and $n^{(i)}$, provided the opacity to emerge from the limb plane to the observer is not much larger than 1 . The parameter $r_{e f f}^{(i)}$ and $n^{(i)}$ can be fixed by using a forward radiative transfer model in order to fit the value of $I / F^{(i)}$. At each altitude, the values of $r_{e f f}^{(i)}$ and $n^{(i)}$ is retrieved using a procedure (LevenbergMarquardt technique) which minimizes the difference between the model results and the observation. We have chosen two quantities to fit; the radiance factor at $250 \mathrm{~nm}$ and the Angström factor which gives the spectral behavior of the radiance factor at each altitudes (displayed in Figure 1).

[17] Thus we are able to retrieve the properties at each level by scanning the atmosphere from top to bottom. Using a constant factor $\rho$ at any altitude to account for the multiple scattering allows to use this procedure even for the multiple scattering solution. Most of the profiles actually have an upper part where the Angström factor is about $\alpha=-4$, and with a radiance factor following an exponential with a quite constant scale height. We assume that this upper part of the profiles are actually due to the atmospheric Rayleigh scattering. Although we sometimes find minor discrepancies, predictions from the MGCM of the Laboratoire de Météorologie Dynamique [Lewis et al., 1999] is consistent with this assumption. Inside the particle layers (due to dust or water ice) we neglect the atmosphere scattering, since particles dominate the scattering process.

\section{Results and Discussion}

\subsection{Scattering by Particles}

[18] The limb scattering intensity depends primarily on the scaled scattering coefficient $\beta \omega P\left(\theta_{s}\right)$ (see equation (4)). The spectral behavior of the radiance factor is then tightly linked to the spectral behavior of this coefficient. In Figure 2, the Angström factor for the scaled scattering coefficient is plotted as a function of the radius, for particles with water ice optical properties [Warren, 1984] and with Mars dust optical properties [Ockert-Bell et al., 1997]. We have used the semi-empirical model of Pollack and Cuzzi [1980] for irregular particles, modified by Showalter et al. [1992]. Two parameters are needed to tune the phase function, $\theta_{\min }$ which set the scattering angle where the phase function reaches its minimum and the slope $S L P$ of the phase function at the 0 scattering angle, $(S L P=-d \log$ $\left.P(\theta) /\left.d \theta\right|_{\theta=0}\right)$. We set them to $120^{\circ}$ and $80 /{ }^{\circ}$ respectively. We show at the end of this paper that these parameters do not significantly modify the results of our analysis. The spectral behavior of $\beta \omega P\left(\theta_{s}\right)$ depends on both the phase angle and on the effective radius of the distribution. The behavior of the spectral slope $\alpha$ with the radius depends on the material optical properties. In particular, the spectral behavior at the limit of small particles is different for dust and water ice optical constants. But for a given material, uncertainties in the refractive indices, even by a factor as large than 2 , do not modify the inferred particle radius.

[19] Most of the observations are taken at phase angle smaller than $60^{\circ}$. Figure 2 shows that in these cases, the Angström factor has a simple monotonic behavior between about $10^{-8}$ and $510^{-7}$ meter. Beyond $10^{-6}$ meter, a single Angström coefficient is no longer sufficient to describe the particle behavior; the quantity $\log \left(\beta \omega P\left(\theta_{s}\right)\right)$ is no longer linear with $\log (\lambda)$. With such a limitation, and also with the 

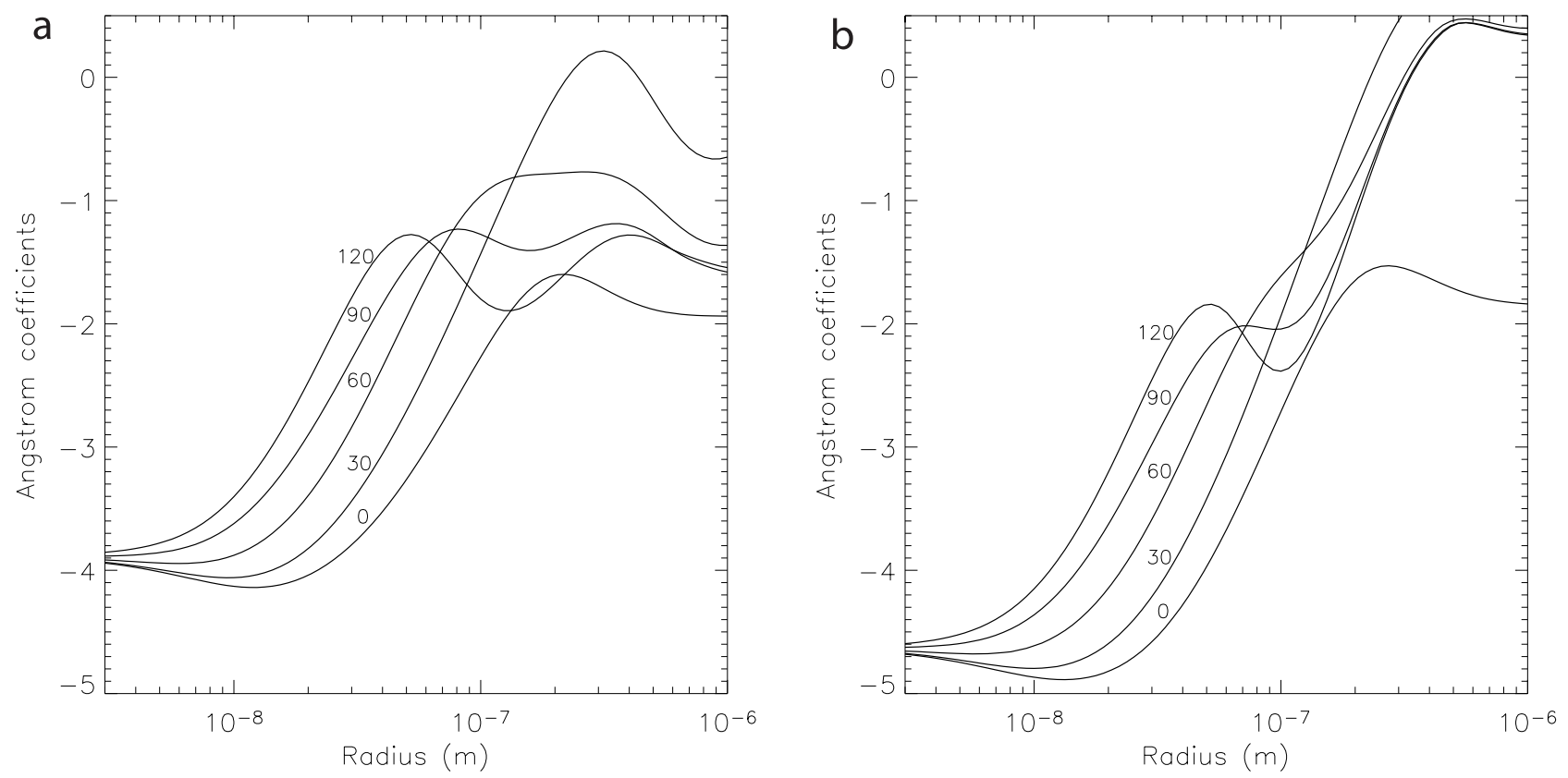

Figure 2. Angström factor as defined in equation (3) as a function of the particle distribution effective radius. Here we assume a log-normal distribution with an effective variance of $v=0.3$ [Hansen and Travis, 1974]. We have computed the values of $\beta \omega P(\theta)$ as a function of the wavelength, and then fitted it with a first-order polynomial to get the slope $\alpha$. For radii larger than $1 \mu \mathrm{m}$, the spectral behavior can no more be fitted with a simple line, and are not shown. The five curves correspond to different phase angles between 0 and $120^{\circ}$. Figure 2a is for dust particle [Ockert-Bell et al., 1997], and Figure $2 \mathrm{~b}$ is for water ice [Warren, 1984].

fact that the radiance factor at the line of sight crossing the limb plane at altitude $z$ is not sensitive to the atmosphere properties at altitude $z$, for low altitude, we know that only the upper part of the dust and cloud layer (that is, when the tangential optical depth is smaller than unity) will be retrieved in our analysis. This limit obviously depends on the atmosphere content, and primarily, on the dust loading.

\subsection{Retrieved Aerosol Properties}

[20] Figure 3 shows profiles with the particle distribution effective radius $r_{\text {eff }}$ as a function of the altitude, along with the scaled scattering coefficient $\beta \omega P\left(\theta_{s}\right)$. We only display here profiles where dust and clouds appear. It is sometimes difficult to separate atmospheric Rayleigh scattering from particle scattering, especially when the Angström factor is low (about -4 ). We believe that further cross comparisons between UV SPICAM observations and Visible observations by OMEGA will be very helpful in discriminating the scattering by various components.

[21] In our results, we generally find an upper profile with an exponential part, generally due to the atmosphere itself. Below a given level, the Angström factor increases rapidly, revealing the presence of dust or water ice particles larger than $10 \mathrm{~nm}$. Several profiles show multiple discrete layers of particles with radius between 10 and $100 \mathrm{~nm}$. It is not possible to determine the composition of the particles, but at these altitudes, such layers are probably made of water ice particles. In this work, we find that both the dust particles and the cloud particles have radii of several tens of nanometers. Cloud particles in detached layer are generally slightly larger than surrounding dust particles by a factor no larger than 2 . The number of particles is generally in the range of $10^{8}$ to $10^{10} \mathrm{~m}^{-3}$. We also find, associated with detached layers, a decrease in the number of particles which can reach one decade.

\subsection{Sensitivity of the Results}

[22] In order to estimate the robustness of our results, despite the numerous uncertainties encountered in this analysis, we perform some sensitivity runs. Our purpose is to test our results against variations of the less well constrained parameters. Figure 4 shows the retrieval for the orbit 641 . We selected this data for the test because a marked detached layer appears, and because the phase angle is $100^{\circ}$, a value that maximizes the sensitivity to the tuning parameter of the semi-empirical model of scattering, which is more relevant to the side scattering rather than the backscattering. We have modified the multiple scattering factor, the semi-empirical model parameters $\theta_{\min }$ and SLP. The last parameter we changed is the distribution effective $\nu_{\text {eff. }}$ We find that the retrieval of particle radius do not significantly suffer from these uncertainties, and the scaled scattering coefficient can vary by a factor of about 2 . This shows that, although quite simple, produces robust results, able to give a correct view of the cloud and dust layer observed with SPICAM UV.

\subsection{Discussion}

[23] In this study, we find particle radii which significantly differ from other estimates. Our estimate is essentially based on the spectral behavior of particle scattering in the UV. Although limited information available in SPICAM 

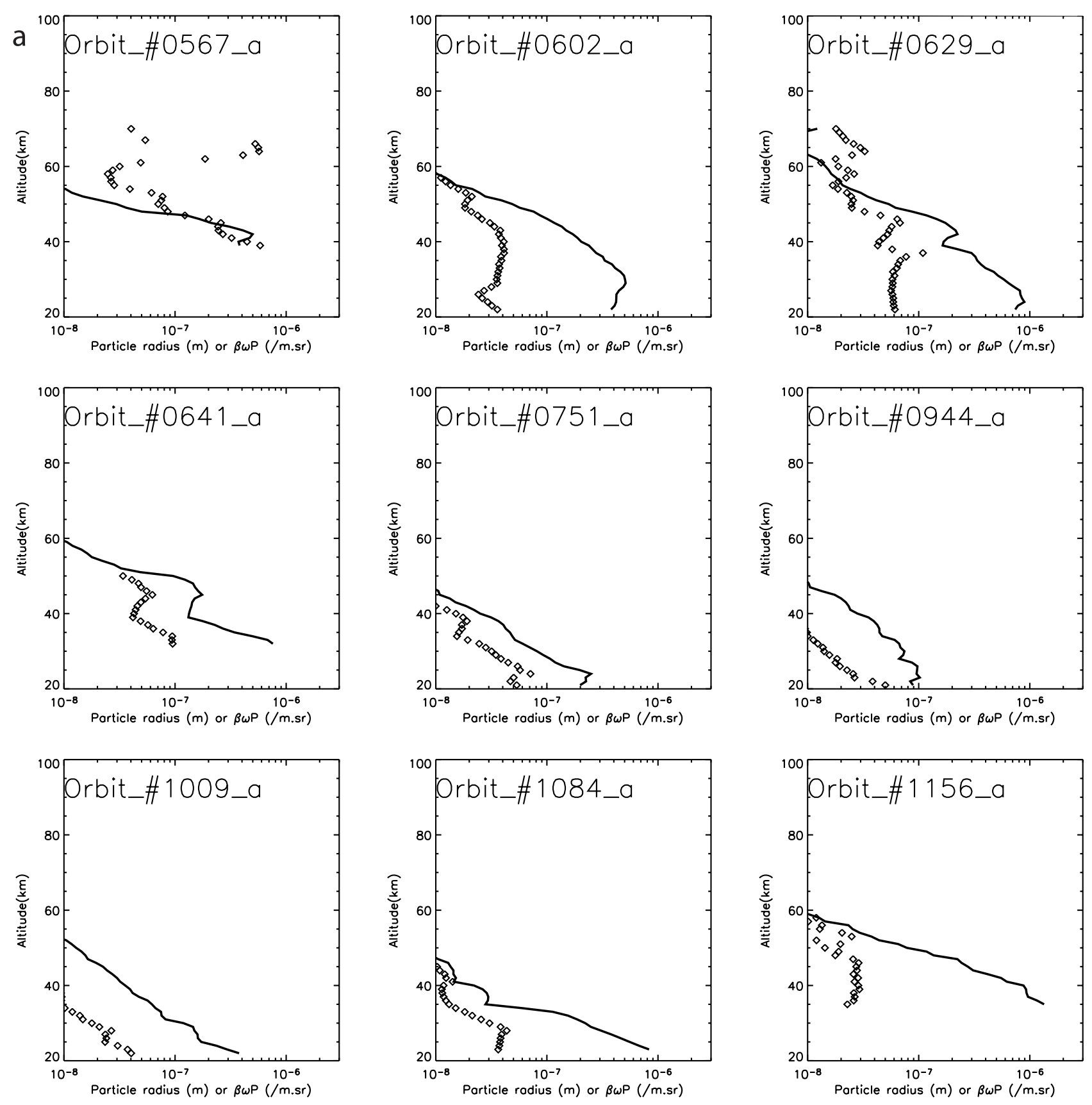

Figure 3. (a) Results of the retrieval procedure. The diamond shapes show the vertical profile of particle radius, and the solid line shows the scaled scattering coefficient $\beta \omega P(\theta)$ at $250 \mathrm{~nm}$ wavelength, where theta is the scattering angle of the observation (that is, $180-\phi$, where $\phi$ is the phase angle). (b) Same as Figure 3a. (c) Same as Figure 3a.

UV spectral domain prevents from deciding on the composition of the fine aerosol component, we speculate that the detached layers are condensation clouds, formed from $\mathrm{H}_{2} \mathrm{O}$ ice (taking into account corresponding thermal conditions in the atmosphere). In previous works, dust particle radii was generally estimated to be in the micrometric range [Pollack et al., 1979, 1995; Tomasko et al., 1999; Markiewicz et al., 1999; Clancy et al., 2000, 2003; Korablev et al., 2005]. Yet, it is not discrepant. These works concerned data probing the dust layer from the ground or from orbit, embracing the whole layer in one view.
[24] As we noted above, previous UV photometric measurements made by Mariner 9 [Pang et al., 1976; Chýlek and Gramm, 1978] and polarization measurements from ground-based observations [Dollfus et al., 1974; Dlugach et al., 2002] were used to infer particle properties. It was found that photometric and polarimetric observation as a function of the phase angle, depends on hypothesis about the particle structure [Dlugach et al., 2002]. In the same way, Pang et al. [1976] fitting the backscattering peak of the dust phase function with Mie theory found micron-sized particles. Later, Chýlek and Gramm [1978] using a model of 

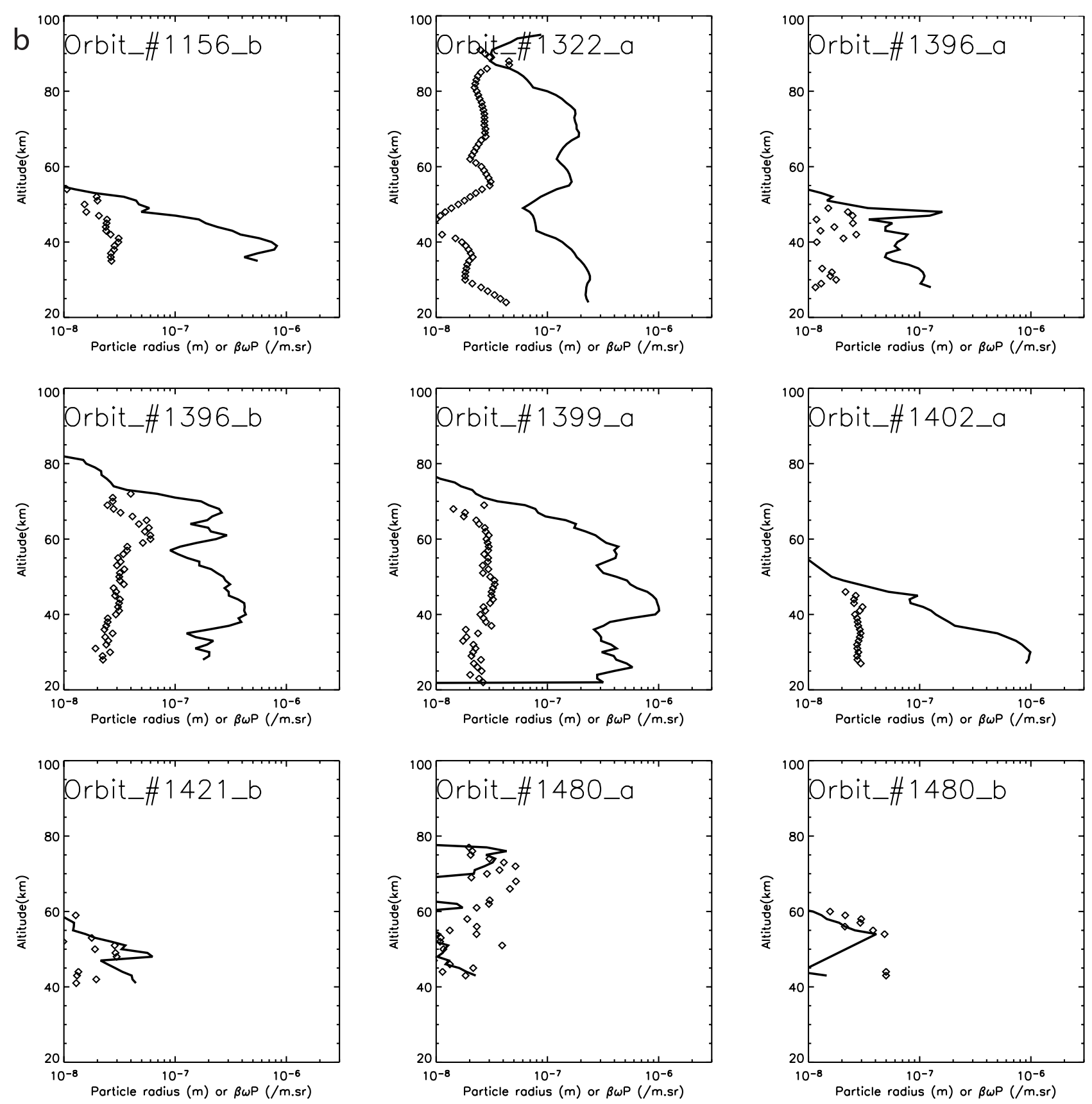

Figure 3. (continued)

scattering by irregular particles, but using a composite phase function (from two data sets taken at two separate sites) found submicronic particle radii. Several remarks may be made about these results: first, Mariner 9 observed Mars during a global dust storm, then large aerosols are expected to be aloft at that time. Second, the composite nature of the data may have introduced bias in Chýlek and Gramm [1978] since the two data sets were taken at two separate places. The two parts of the phase function could not form a consistent set. Finally, side scattering photometry and polarimetric data, especially at short wavelength, are rather sensitive to the size of the subscale structures of the particles than to the size of the particle itself. The latter case also occurred about Titan aerosols that was actually shown to be aggregated particles rather than spheres [West and Smith, 1991]. Then, UV phase function or polarization function may tell more about the particle structure than on the particle size. Our results are primarily based on spectral behaviors (Angström factors) of scattering cross sections, and not to phase effects. These quantities are willing to yield robust conclusions about particle size, especially concerning small particles which spectral behaviors have well marked characteristics (Figure 2).

[25] From a microphysical point of view, it is expected to observe a gravitational segregation of the aerosols. Particle settling speed is roughly proportional to the radius, thus 

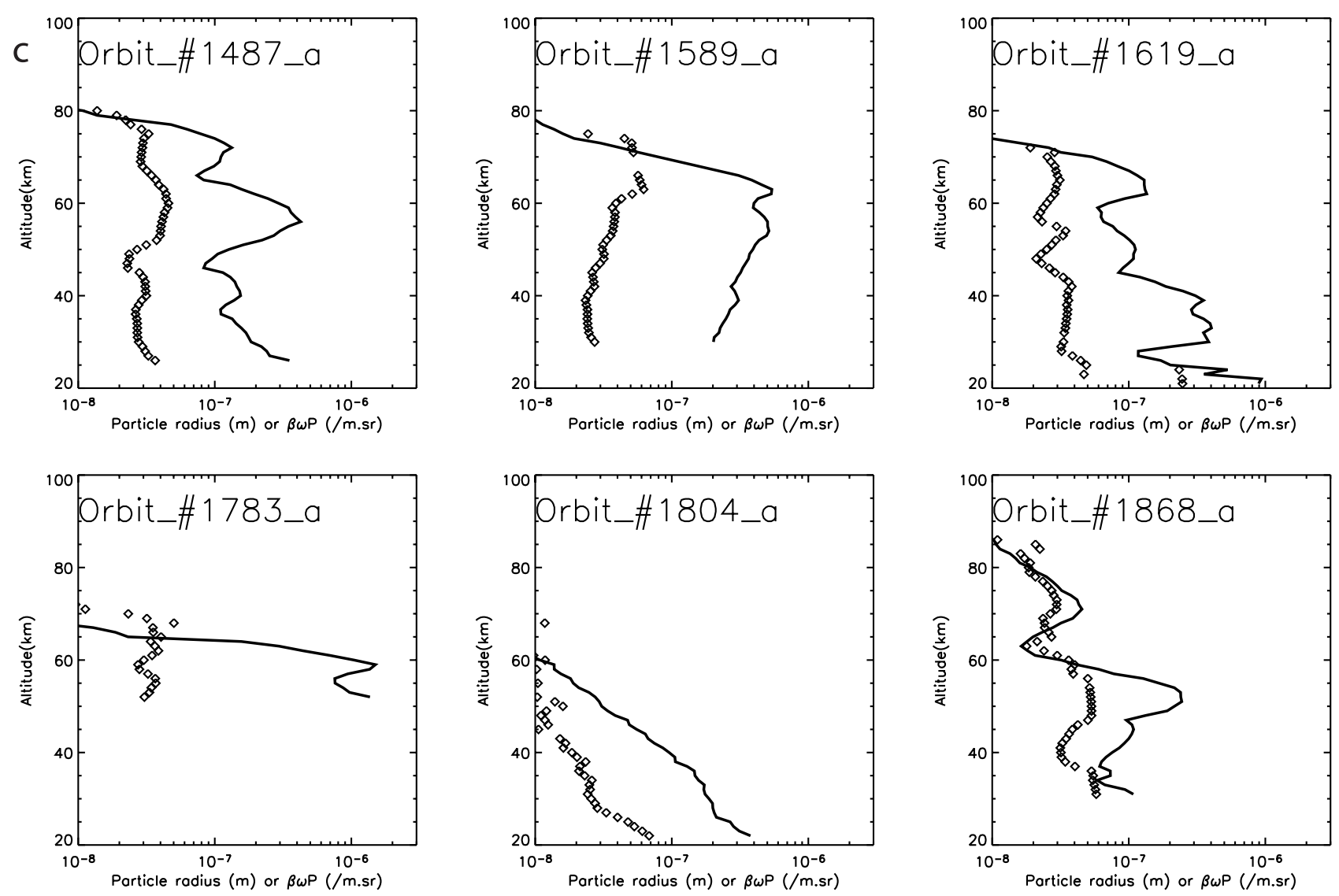

Figure 3. (continued)
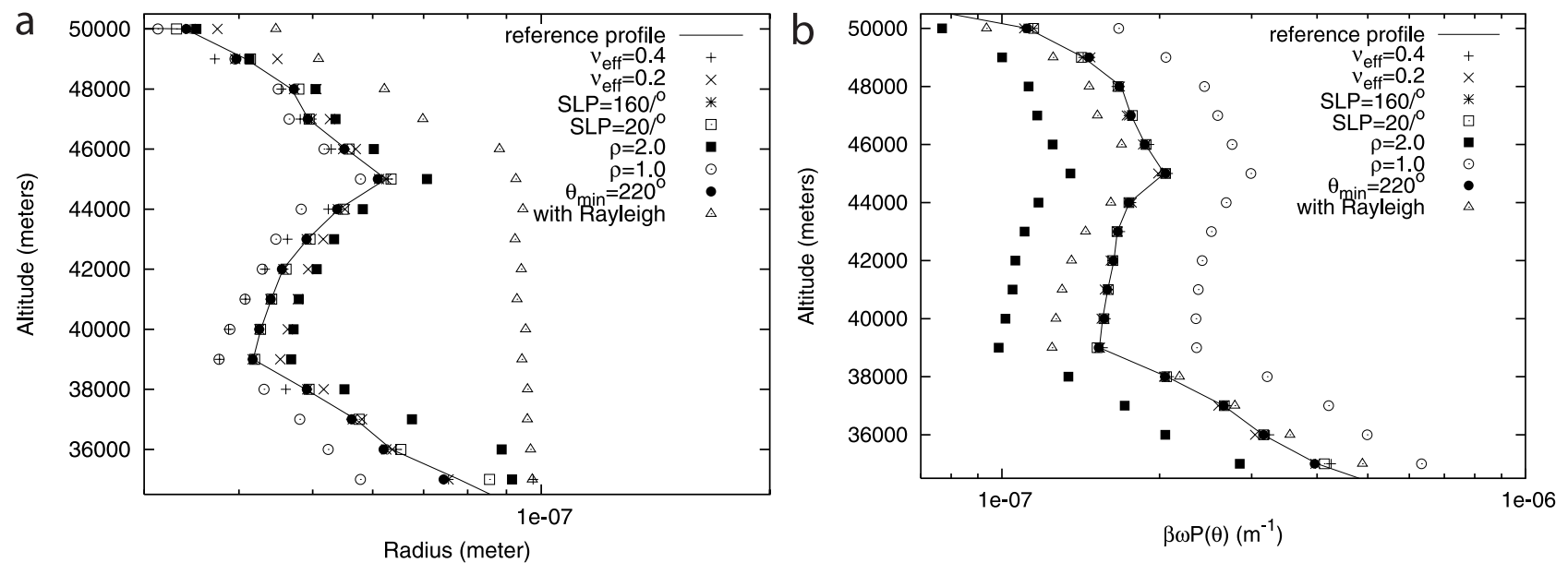

Figure 4. Close-up on the result of the orbit 641. The curves show (a) the radius and (b) the scaled scattering coefficient retrieved for the reference set parameters and for modified parameters. We have changed the effective variance $(\nu=0.1$ and $\nu=0.6$ instead of $\nu=0.3$ for the reference profile), the phase function slope $S L P$, and the angle at the minimum of the phase function $\theta_{\min }$ of Pollack and Cuzzi [1980] model $\left(S L P=20 /{ }^{\circ}\right.$ and $160 /{ }^{\circ}$ instead of $80 /{ }^{\circ}$ and the $\theta_{\min }=220^{\circ}$ instead of $120^{\circ}$. The last test consisted in using the temperature and pressure profile from the Mars GCM database [Lewis et al., 1999] to include the atmosphere Rayleigh scattering in the forward model. We find that most of the parameters have only a small effect on the radius profile and may change the scaled scattering coefficient by a factor of about 2 . Including the effect of the atmosphere from a realistic profile would change the radius retrieval by a factor of 2 . Although the effect exists, it does not deeply change our results. 
whatever is the physical process which lifts dust upward (e.g., advection or mixing), small particles are transported upward more easily than larger one, and distribution scale height is conversely related to the aerosol radii. This effect have been modeled [Chassefière et al., 1992; Korablev et al., 1993; Montmessin et al., 2002] and results consistently show a decrease in effective particle radii. Small particles at high altitude could be consistent with some broad particle size distributions used to fit Mars data [Clancy et al., 1995]. However, this type of wide distribution is not stable against coagulation [Montmessin et al., 2002]. Generally, segregation effect is not sufficient to explain particles as small as observed and Montmessin et al. [2002] show that bimodal distributions (a classical 1.5- $\mu \mathrm{m}$ mode and a fine particles $0.1-0.2 \mu \mathrm{m}$ mode) are needed to explain the presence of small particle $(200 \mathrm{~nm})$ above $30 \mathrm{~km}$ as observed in some Viking limb scattering profiles [Jaquin et al., 1986].

[26] The presence of such fine component of Mars aerosol is consistent with the condensate properties of Mars clouds [Montmessin et al., 2002]. It is important because the water cycle on Mars is concerned. If a large number of condensation nuclei is available in the atmosphere, a significant supersaturation never occurs: whenever temperature in the atmosphere falls below saturation, water vapor condensates to form ice clouds. When we observe detached layers, and interpret them as water ice clouds, the derived size of ice particles is about twice as large as that of the dust particles. We can compare this result with the result of Chassefière et al. [1992], who derived cloud particles with $r_{\text {eff }}=0.3-0.6 \mu \mathrm{m}$ from Phobos-88 solar occultation measurements.

\section{Conclusion}

[27] In this work, we have presented some observations made at the limb of Mars by the SPICAM UV spectrometer on Mars Express. The spectral domain of 220-300 nm was analyzed. Rayleigh scattering by the $\mathrm{CO}_{2}$ atmosphere generally dominates the upper part of these profiles (above about $50 \mathrm{~km}$ ). Pronounced increases in the scattering, associated with sharp increases in Angström factors, in the lower part of the atmosphere are interpreted in terms of large concentration of very small particles. Several profiles show detached layers which are probably cloud layers, although information is not sufficient to discriminate between ice and dust. Our analysis show that both dust and cloud particles have sizes between 10 and $100 \mathrm{~nm}$. We also find that particle in detached layers (possibly cloud particles) are generally two or three times larger than the dust particles in the surrounding environment. The values found in this work are consistent with estimates made from star occultations in UV with SPICAM [Montmessin et al., 2006a, 2006b].

[28] Acknowledgments. Mars Express is a space mission from ESA (European Space Agency). We wish to express our gratitude to all ESA members who participated in this successful mission. We thank also Astrium for the design and construction of the spacecraft. We thank our collaborators at the three institutes for the design and fabrication of the instrument (Service d'Aronomie/France, BIRA/Belgium, and IKI/Moscow). We wish to thank CNRS and CNES for financing SPICAM in France. We wish to thank the Space Division of the Belgian Federal Science Policy Office for supporting this project through the ESA PRODEX program. The Russian team acknowledges support of RFFI grant 04-0216856-a.

\section{References}

Bertaux, J. L., et al. (2000), The study of the Martian atmosphere from top to bottom with SPICAM light on Mars express, Planet. Space Sci., 48, $1303-1320$

Bertaux, J. L., et al. (2005), Global structure and composition of the Martian atmosphere with SPICAM on Mars express, Adv. Space Res., 35(1), $31-36$.

Bertaux, J.-L., et al. (2006), SPICAM on board Mars Express: Instrument, operations, observing modes, and overview of typical results, J. Geophys. Res., doi:10.1029/2006JE002690, in press.

Chassefière, E., J. E. Blamont, V. A. Krasnopolski, O. I. Korablev, S. K Atreya, and R. A. West (1992), Vertical structure and size distributions of Martian aerosols from solar occultation measurements, Icarus, 97, 4669.

Chýlek, P., and G. M. Gramm (1978), Scattering by nonspherical particles and optical properties of Martian dust, Icarus, 36, 198-203.

Clancy, R. T., S. W. Lee, G. R. Gladstone, W. McMillan, and T. Roush (1995), A new model for Mars atmospheric dust based upon analysis of ultraviolet through infrared observations from Mariner 9, Viking, and Phobos, J. Geophys. Res., 100, 5251-5263.

Clancy, R. T., et al. (2000), An intercomparison of ground-based millimeter, MGS TES and Viking atmospheric temperature measurements: Seasonal and interannual variability of temperatures and dust loading in the global Mars atmosphere, J. Geophys. Res., 104, 9553-9571.

Clancy, R. T., M. J. Wolff, and P. R. Christensen (2003), Mars aerosol studies with the MGS TES emission phase function observations: Optical depths, particle sizes, and ice cloud types versus latitude and solar longitude, J. Geophys. Res., 108(E9), 5098, doi:10.1029/2003JE002058.

Dlugach, Z. M., M. I. Mishchenko, and A. V. Morozhenko (2002), The effect of the shape of dust aerosol particles in the Martian atmosphere on the particle parameters, Sol. Syst. Res., 36, 367-373.

Dollfus, A., J. M. Dlugach, A. V. Morozhenko, and E. G. Yanovitskii (1974), Optical parameters of the atmosphere and surface of Mars: II, Dust storms, Astron. Vestn., 8, 211-222.

Hansen, J. E., and L. D. Travis (1974), Light scattering in planetary atmospheres, Space Sci. Rev., 16, 527-610.

Jaquin, F., P. Gierasch, and R. Kahn (1986), The vertical structure of limb hazes in the Martian atmosphere, Icarus, 68, 442-461.

Korablev, O. I., V. A. Krasnopolsky, A. V. Rodin, and E. Chassefière (1993), Vertical structure of Martian dust measured by solar infrared occultations from the PHOBOS spacecraft, Icarus, 102, 76-87.

Korablev, O., V. I. Moroz, E. V. Petrova, and A. V. Rodin (2005), Optical properties of dust and the opacity of the Martian atmosphere, Adv. Space Res., 35(1), 21-30.

Lewis, S. R., M. Collins, P. L. Read, F. Forget, F. Hourdin, R. Fournier, C. Hourdin, O. Talagrand, and H.-P. Huot (1999), A climate database for Mars, J. Geophys. Res., 104, 24,177-24,194.

Markiewicz, W. J., R. M. Sablotny, H. U. Keller, N. Thomas, D. Titov, and P. H. Smith (1999), Optical properties of the Martian aerosols as derived from Imager for Mars Pathfinder midday sky brightness data, J. Geophys. Res., 104, 9009-9018.

Montmessin, F., P. Rannou, and M. Cabane (2002), New insights into Martian dust distribution and water-ice cloud microphysics, J. Geophys. Res., 107(E6), 5037, doi:10.1029/2001JE001520.

Montmessin, F., et al. (2006a), Sub-visible $\mathrm{CO}_{2}$ ice clouds detected in the Martian mesosphere, Icarus, 183, 403-410, doi:10.1016/j.icarus.2006. 03.015 .

Montmessin, F., E. Quémerais, J.-L. Bertaux, O. Korablev, A. Federova, P. Rannou, and S. Lebonnois (2006b), Stellar occultation at UV wavelengths by the SPICAM instrument: Retrieval and analysis of Martian haze profiles, J. Geophys. Res., 111, E09S09, doi:10.1029/2005JE002662.

Morozhenko, A. V. (1974), Optical parameters of the atmosphere and surface of Mars: I. Aerosol composition of clear atmosphere, Astron. Vestn., $8,121-127$.

Ockert-Bell, M. E., J. F. Bell, J. B. Pollack, C. P. McKay, and F. Forget (1997), Absorption and scattering properties of the Martian dust in the solar wavelengths, J. Geophys. Res., 102, 9039-9050.

Pang, K., and J. M. Ajello (1977), Complex refractive index of Martian dust-Wavelength dependence and composition, Icarus, 30, 63-74.

Pang, K., J. M. Ajello, C. W. Hord, and W. G. Egan (1976), Complex refractive index of Martian dust: Mariner 9 ultraviolet observations, Icarus, 27, 55-67.

Pollack, J. B., and J. N. Cuzzi (1980), Scattering by nonspherical particles of size comparable to wavelength-A new semi-empirical theory and its application to tropospheric aerosols, J. Atmos. Sci., 37, 868881 . 
Pollack, J. B., D. S. Colburn, F. M. Flasar, R. Kahn, C. E. Carlston, and D. G. Pidek (1979), Properties and effects of dust particles suspended in the Martian atmosphere, J. Geophys. Res., 84, 2929-2945.

Pollack, J. B., M. E. Ockert-Bell, and M. K. Shepard (1995), Viking Lander image analysis of Martian atmospheric dust, J. Geophys. Res., 100, $5235-5250$

Quémerais, E., J.-L. Bertaux, O. Korablev, E. Dimarellis, C. Cot, B. R Sandel, and D. Fussen (2006), Stellar occultations observed by SPICAM on Mars Express, J. Geophys. Res., 111, E09S04, doi:10.1029/ 2005JE002604.

Rages, K., and J. B. Pollack (1983), Vertical distribution of scattering hazes in Titan's upper atmosphere, Icarus, 55, 60-62.

Rannou, P., M. Cabane, R. Botet, and E. Chassefière (1997), A new interpretation of scattered light measurements at Titan's limb, J. Geophys. Res., 102, 10,997-11,014.

Showalter, M. R., J. B. Pollack, M. E. Ockert, L. R. Doyle, and J. B. Dalton (1992), A photometric study of Saturn's F Ring, Icarus, 100 $394-411$.
Smith, M. D. (2004), Interannual variability in TES atmospheric observations of Mars during 1999-2003, Icarus, 167, 148-165.

Tomasko, M. G., et al. (1999), Properties of dust in the Martian atmosphere from the imager on Mars Pathfinder, J. Geophys. Res., 104, 8987-9007.

Tran, T.-T., and P. Rannou (2004), Comparing 3D spherical Monte-Carlo and 2-stream parallel plane simulation of far-field backscattering image of Titan, Notes Pôle Planétol. 2, Inst. Pierre-Simon Laplace, Paris.

Warren, S. G. (1984), Optical constants of ice from the ultraviolet to the microwave, Appl. Opt., 23, 1206-1225.

West, R. A., and P. H. Smith (1991), Evidence for aggregate particles in the atmospheres of Titan and Jupiter, Icarus, 90, 330-333.

J.-L. Bertaux, F. Montmessin, S. Perrier, P. Rannou, and A. Rébérac, Service d'Aéronomie du CNRS, Université de Versailles St-Quentin, BP3, F-91371 Verrières le Buisson, France. (pra@aerov.jussieu.fr)

O. Korablev, Space Research Institute (IKI), 84/32 Profsoyuznaya, 117810 Moscow, Russia. 